\title{
Qualification for Product Development
}

\author{
Weiqiang Wang, Michael H. Azarian and Michael Pecht \\ Center for Advanced Life Cycle Engineering (CALCE) \\ University of Maryland \\ College Park, MD 20742, USA \\ Email: pecht@calce.umd.edu; \\ Telephone number: (01)301-405-5323; \\ Fax number: (01)301-314-9269
}

\begin{abstract}
The aim of qualification is to verify whether a product meets or exceeds the reliability and quality requirements of its intended application. Qualification plays an important role in the process of product development. It can be classified by its specific purpose at different stages of the product development process. In this paper, a new methodology of product qualification is proposed based on physics-of-failure. This methodology consists of: product configuration and material information collection; application requirement information collection; strength limits and margins; failure modes, mechanisms and effects analysis; definition of qualification requirements; qualification test planning; testing; failure analysis and verification; and quality and reliability assessment. This approach to qualification ensures that it successfully addresses the failure mechanisms applicable to the product's specific design, manufacture, and application conditions.
\end{abstract}

\section{Introduction}

Qualification is the process of demonstrating that an entity or process is capable of meeting or exceeding the specified requirements [1]. Qualification includes activities which ensure that the nominal design and manufacturing process will meet or exceed the specified targets. The purpose of qualification is to obtain the acceptable range of variability for all critical product parameters affected by design and manufacturing, such as geometric dimensions and material properties. Attributes that fall outside the acceptable range are termed as "defects" because they have the potential to make the product fail to meet the specified requirements [2].

Qualification can be used in process development and product development. Process qualification involves a set of procedures which validate that a process used to manufacture a product meets specified performance requirements [3]. It is used to provide assurance that a particular process is under control and known to produce qualified products [4]. Product qualification aims to evaluate performance of products under specified operating and environmental conditions within a specified period of time, which will be the focus of this paper.

The performance of a product consists of quality (which includes function) and reliability, whose requirements are set during product design. The qualification process is intended to examine whether the products' performance can meet the design requirements. The product qualification includes the verification of functions, the assessment of reliability in application conditions, and the validation in the system application if the product is a component of a system.

Product qualification can be used to baseline the design, materials and processes. It determines the product performance degradation under normal application conditions. It can also be used to compare different designs to help make design decisions. Product qualification is used to meet the requirements of customers with consideration of the intended application and application conditions.

\section{Qualification in product development process}

Qualification occurs in different stages of the product development process, as shown in Figure 1. Qualification activities in different stages have different purposes. Virtual qualification is to evaluate the functional and reliability performance of the product design without any physical testing on the product. Virtual qualification involves using computer-assisted modeling and simulation based on physics-of-failure (PoF) [5]. Product qualification is to evaluate the product based on the physical testing on the manufactured prototype. The purpose is to verify whether the product has met or exceeded its intended quality and reliability requirements. After virtual and product qualification, the products are mass produced. During and after the manufacturing process, the products can be inspected and tested to evaluate their quality and defected parts can be screened out. This process can be considered as a third stage in the overall qualification process, and it is more commonly referred to as quality assurance testing.

Virtual and product qualification efforts are part of a larger process of product design and development. At various intersections of the process, maturity levels can be assigned to indicate progress and specific readiness for the next phase. The design and product qualification process may include feedback iterations shown in Figure 2. If the product design is found to be unqualified during the virtual qualification process, it is modified and then virtually requalified before proceeding to the next phase. Similarly, when a design has successfully passed through the virtual qualification process, but does not meet the qualification requirements during product qualification stage, feedback iterations may be necessary. In this case, the virtual qualification process and specifically, the physics-offailure based models may have to be re-evaluated and modified. After design completion, the product is manufactured in high volume and subjected to quality assurance testing during and after the process. 


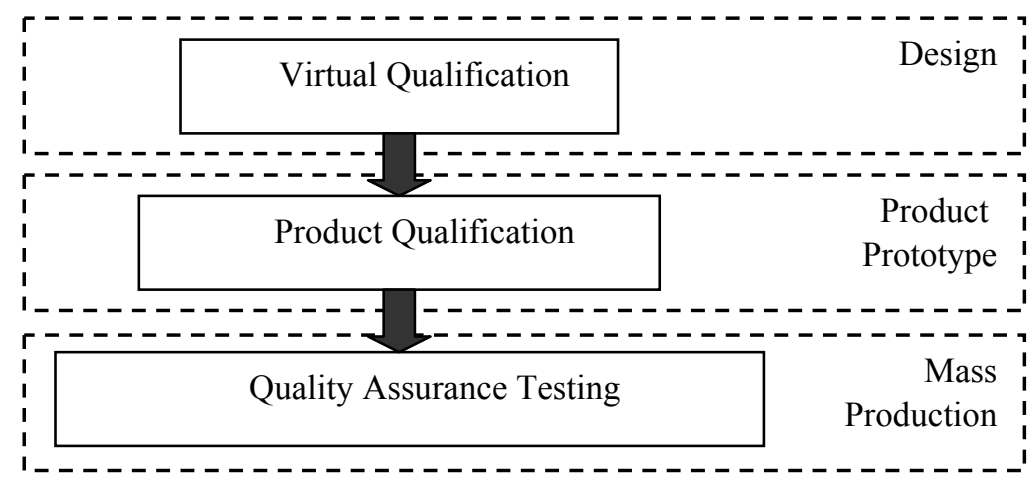

Figure 1: Qualification activities in different stages of a product development process

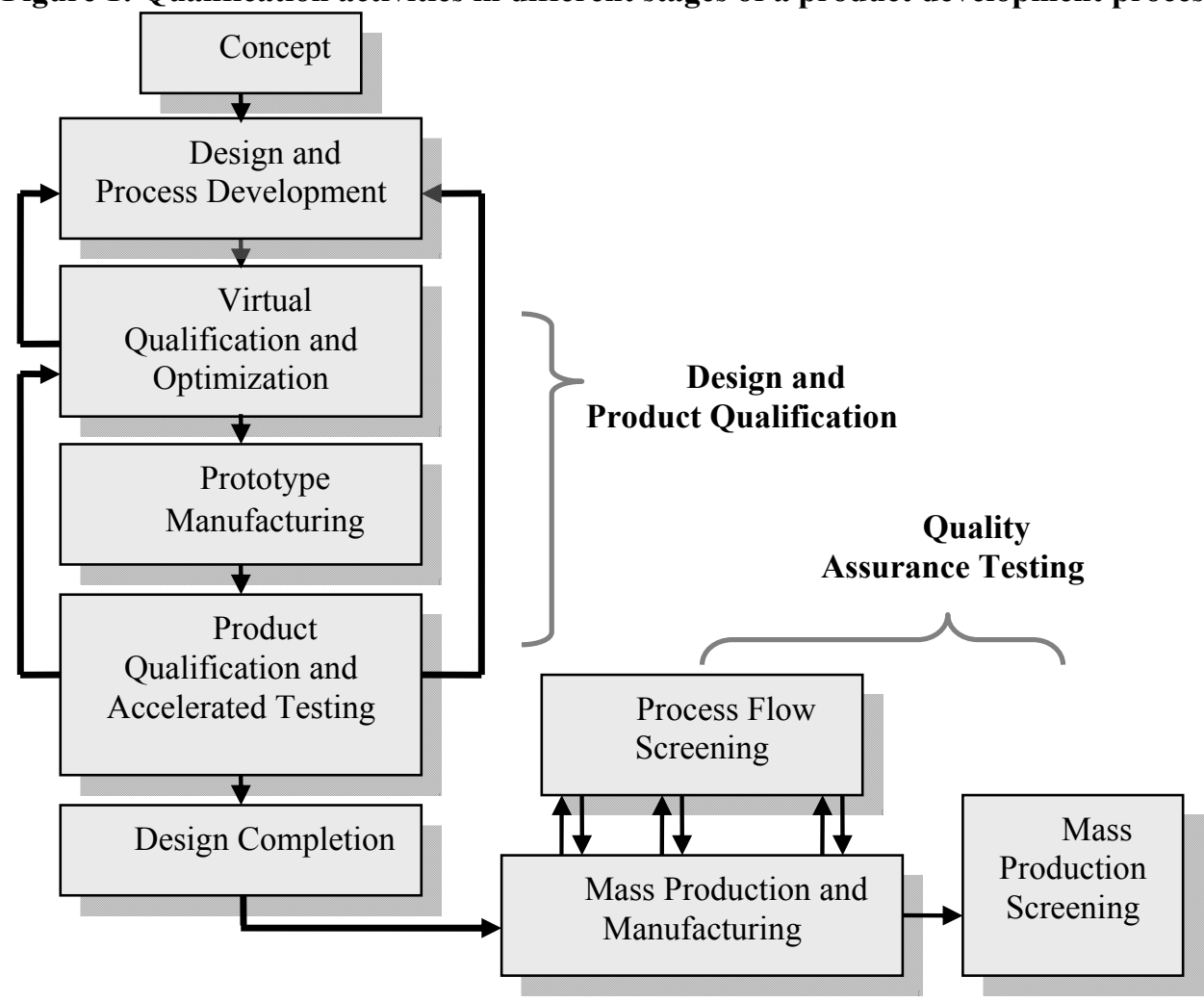

Figure 2: Qualification and quality assurance testing within the product design and manufacturing process flow including iterative feedback process

The objectives of qualification testing are to (a) evaluate the quality of a product to see if it meets the design requirements, (b) develop information on the integrity of a product and its structure, (c) estimate the expected service life and reliability and (d) evaluate the effectiveness of materials, processes, and designs,. Qualification tests estimate expected life and design integrity of a product. Most tests are not conducted under the normal application conditions, but at accelerated levels of stresses to accelerate potential failure mechanisms at associated sites in a product.

Successful qualification of a sampling of a product does not assure that all products made by the same manufacturer to the same specifications will also meet the qualification requirements. Qualification should be conducted by the manufacturer, although the customer may do so for special applications. Data from all possible sources should be used in qualification. These sources include material and component suppliers' test data, qualification data from similar items, and accelerated test data from materials, components, and subassemblies.

\subsection{Virtual qualification}

Virtual qualification is a methodology for assessing and improving the reliability of products through the use of validated failure models and simulation tools [6] [7]. It is also an important step in developing effective physical tests to verify product reliability. The application of virtual qualification has led to significant cost savings for commercial and military organizations.

Virtual qualification is the first stage of the overall qualification process. It is the application of PoF based reliability assessment to determine if a proposed product can survive its anticipated life cycle [8]. Virtual qualification (also called simulation-assisted reliability assessment) assesses whether a part or system can meet its reliability goals under anticipated life cycle profiles based on its materials, geometry, and operating characteristics. The technique involves the application of 


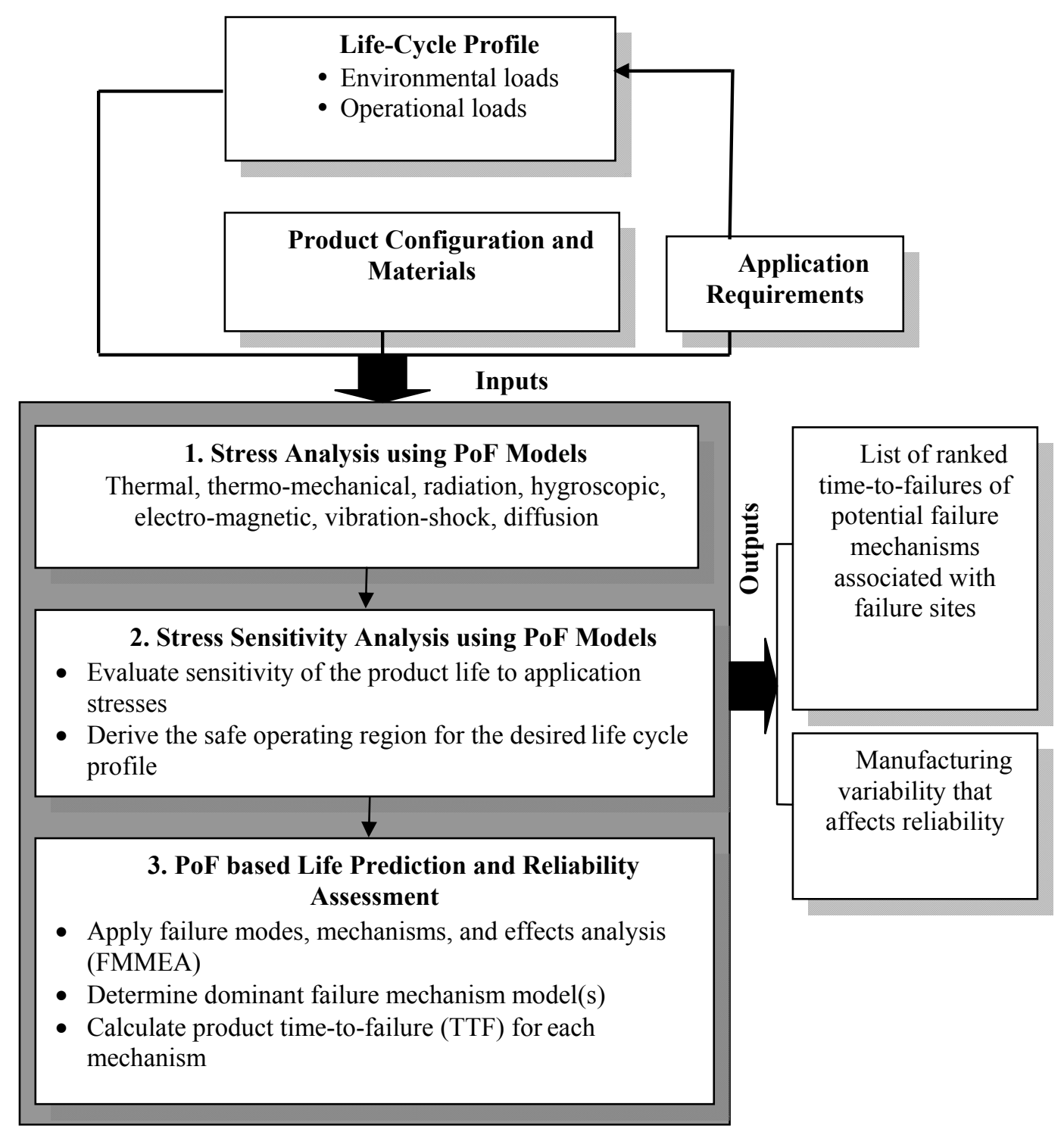

Figure 3: Flowchart of virtual qualification

simulation software to model physical hardware to determine the probability of the system's meeting desired life goals [1] [9] [10] [11].

Engineers can realize significant time savings by developing a flow-through process of life-cycle characterization, product modeling, load transformation, and failure assessment to qualify products. Virtual qualification can be applied at design stages and hence it helps move reliability assessment process into the design phase [6] [7]. It allows the design team to consider qualification at the initial stages of design, technology and functional definition, and supplier selection. A flowchart of virtual qualification is shown in Figure 3. This system takes advantage of advances in computer-aided engineering software tools that permit components and systems to be qualified based on an analysis of the susceptibility of their designs to failure due to a number of fundamental physical and chemical mechanisms [12] [13]. The reliability assessment tool assesses the candidate and existing product designs for reliability in many different environments using a database of fully validated PoF models. It calculates time-to-failure of fundamental mechanisms that cause failures and evaluates the effects of different manufacturing processes on reliability by calculating the time-to-failure as a function of typical manufacturing tolerances and defects [14] [15]. It facilitates the selection of cost-effective test parameters for validating reliability assessment and design and also aids the selection of high-volume commercial off-the-shelf components by permitting their virtual qualification [16].

The inputs consist of life cycle profile and product characteristics. The life cycle profile can be further categorized as environmental and operational stresses as shown. The inputs are fed into physics-of-failure (PoF) model and simulation software where stress analysis, reliability assessment and stresses sensitivity analysis are performed. The outputs of virtual qualification are predicted time-to-failures (TTF) based on the most dominant failure mechanisms, stress margin conditions, and screening and accelerated testing conditions.

In addition to time-to-failure prediction and reliability assessment, virtual qualification combined with advanced optimization techniques can be used to optimize the design criteria including cost, electrical performance, thermal 


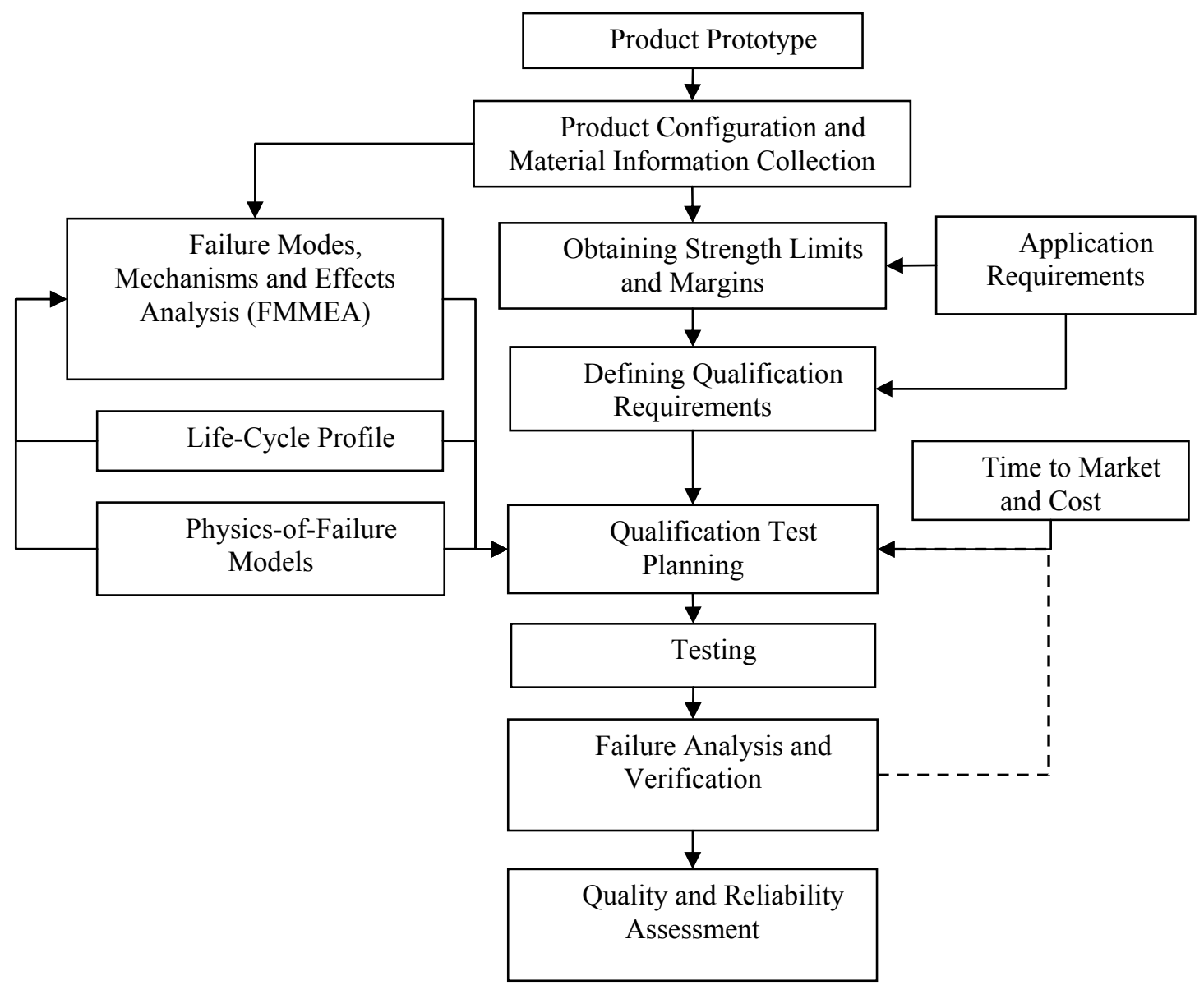

Figure 4: Flowchart of methodology of product qualification

management, physical attributes, and reliability. By examining potential trade-offs between the aforementioned criteria, ideal values can be achieved for specific applications.

In the virtual qualification process, it is imperative to use the most accurate inputs including material properties, design configuration, dimensions, and operational and environmental conditions. Furthermore, the failure mechanism models used in time-to-failure (TTF) prediction and reliability assessment must be valid. If the data or models on which the virtual qualification is performed is inaccurate or unreliable, any qualification results based on the data or models are suspicious.

\subsection{Product qualification}

Product qualification is the evaluation of products after prototype manufacturing. It is intended to qualify a product before its mass production. The qualification includes the verification of their function and performance, the validation in the system application (if applicable) and the qualification for processability and reliability. After the qualification, products' specifications that do not meet the design and customer requirements should be reported to the design team for correction action. Parameters in qualification tests, failure modes and failure mechanisms of products during qualification tests will be provided to the design team as feedback on how to improve the design or the manufacturing process. Product qualification will be the main focus of this paper. It will be detailed in section 3 .

2.3 Quality assurance testing during mass production
The qualification tests that take place during mass production and before the products are shipped to the customer are more properly considered to be quality assurance testing that ensure that the products are manufactured according to the design within allowable tolerances. Furthermore, accelerated stresses are applied to the products to accelerate early failures that are caused by manufacturing defects. These qualification tests ensure the quality of products that will be used in field applications. Manufacturing defects are screened out of the product shipping list.

\section{Methodology of product qualification}

Upon completion of virtual qualification, the product prototype is manufactured, and then product qualification process begins. In the product qualification process, physical tests are applied to the manufactured prototype to verify whether it meets its functionality and reliability requirements. If the design and manufacturing processes that were initially considered during the virtual qualification process has not been modified, then product qualification process essentially begins with strength limit testing or HALT. Conversely, any changes made to the product characteristics outside the design and manufacturing tolerance ranges requires virtual requalification or a product qualification process that includes the re-definition of product characteristics and the repeat of FMMEA process. The center for advanced life cycling engineering (CALCE) developed a methodology for product qualification to make the process of 
qualification clearer to engineers. This methodology includes collecting product configuration and material information, collecting product life cycle profile, strength limit and margins, failure mode, mechanisms and effects analysis (FMMEA), defining qualification requirements, qualification test planning, testing, failure analysis and verification and quality and reliability assessment. A flowchart is shown in Figure 4.

\subsection{Product configuration and materials}

One of the most fundamental steps in the product qualification is to characterize the product in terms of its configuration and materials. Information about the configuration and materials of the product provides engineers with the basic knowledge for the qualification. The information includes the architecture of product, the materials used to manufacture the product and the process that the materials have experienced during the manufacturing process.

A product consists of a number of components and subassemblies working together to deliver the overall function of the product. Each subassembly may consist of lower level assemblies that are also interconnected. The architecture of a product describes the physical and functional relations between the subassemblies. The hardware configuration of the product describes the design of the components and subassemblies and the product architecture. It may also include the effects of the manufacturing processes on the final product in the form of tolerances on the dimensions and material properties.

The hardware of electronic equipments includes electronic parts, printed circuit boards, connectors, and enclosures. An electronic part may be a semiconductor chip and the package that provides power and ground inputs, signal communication paths to the outside and protection from the environment. An electronic part can also be a passive component such as a resistor or capacitor. The part geometry and structure, the sub-component geometry, and the connection methods, such as wirebonds or solder balls, will also be characterized. A printed circuit board description include the materials, layer stacks, the connections between layers, the additions to the layers, such as heat spreaders, and elements like stiffeners.

Materials used to construct a product influence the level of stress on the product due to external and internal loads and the process of damage accumulation [17] [18]. To the extent that materials influence stress and damage, their physical properties should be characterized [19] [20]. For example, a failure in a solder joint may be driven by stress arising from repeated temperature excursions. In this situation, the coefficient of thermal expansion of a material is needed to determine the cyclic stress state. In another situation, a failure may occur due to a reduction in the contact force between connector elements. This situation may require the elastic modulus of the connector elements, loading elements and their housings to determine the contact force and its degradation pattern. Properties for common materials used in electronic products can be found in references [19] [20] [21].

Products are not normally produced by a single manufacturing process. They often require a sequence of different processes to achieve all the required attributes of the final product. The manufacturing process applies stresses on materials, may produce residual stress, and may even modify some of material properties. For example, a lead-free reflow profile can change the thermo-physical properties of a printed circuit board. The variations in geometry and material properties caused by different manufacturing processes need to be characterized.

\subsection{Life-cycle profile}

The second step in the product qualification is to understand the life-cycle profile (LCP) of products. The LCP is the base for selecting product qualification test conditions, including types and severity levels. The major task in understanding the LCP is to characterize the loads applied onto the product during its life cycle. The environmental loading to a component should be considered to be from its surrounding environment as well as from within, but not from the system level environment. For example, when a silicon chip is working, the temperature and humidity of its environment will affect its function and reliability, as does the heat generation within the chip.

A LCP is a time history of events and conditions associated with a product from its release from manufacturing to its removal from service. The life cycle includes various phases that an item will encounter in its life, such as: handling, shipping, and storage prior to use; mission profiles while in use ${ }^{1}$; phases between missions, such as stand-by or storage, transfer to and from repair sites and alternate locations; geographical locations of expected deployment and maintenance; and maintenance and repair procedures for the system and the component.

Loads applied to the product during its life cycle drive the processes that lead to product degradation and failure. The life cycle of a product includes manufacturing and assembling, testing, reworking, storing, transporting and handling, operating (e.g., modes of operation, on-off cycles), and repairing. The life cycle loads include assembly/installation related loads, environmental loads and operational loads. These loads can be thermal [22], mechanical, chemical, physical, and/or operational loading conditions. Various combinations and levels of these loads can influence the reliability of the product. The extent and rate of product degradation depend upon the nature, magnitude and duration of exposure to such loads.

Since a product may experience numerous loads, it is necessary to identify the most critical ones to its function and reliability. Some of the loads will play major roles in activating and accelerating the failure of the product, while others can be ignored. For example, low levels of radiation can often be ignored for ground-based electronic products, since this rarely causes dysfunction or damage to products. Whether the loads can or cannot be ignored depends on the critical failure mechanisms that are identified in the analysis, which consider the life cycle conditions.

\footnotetext{
${ }^{1}$ In some cases, the environmental factors experienced by constituents of the product begin before manufacturing -e.g., storage of parts (material) far in advance of their use in manufacturing.
} 


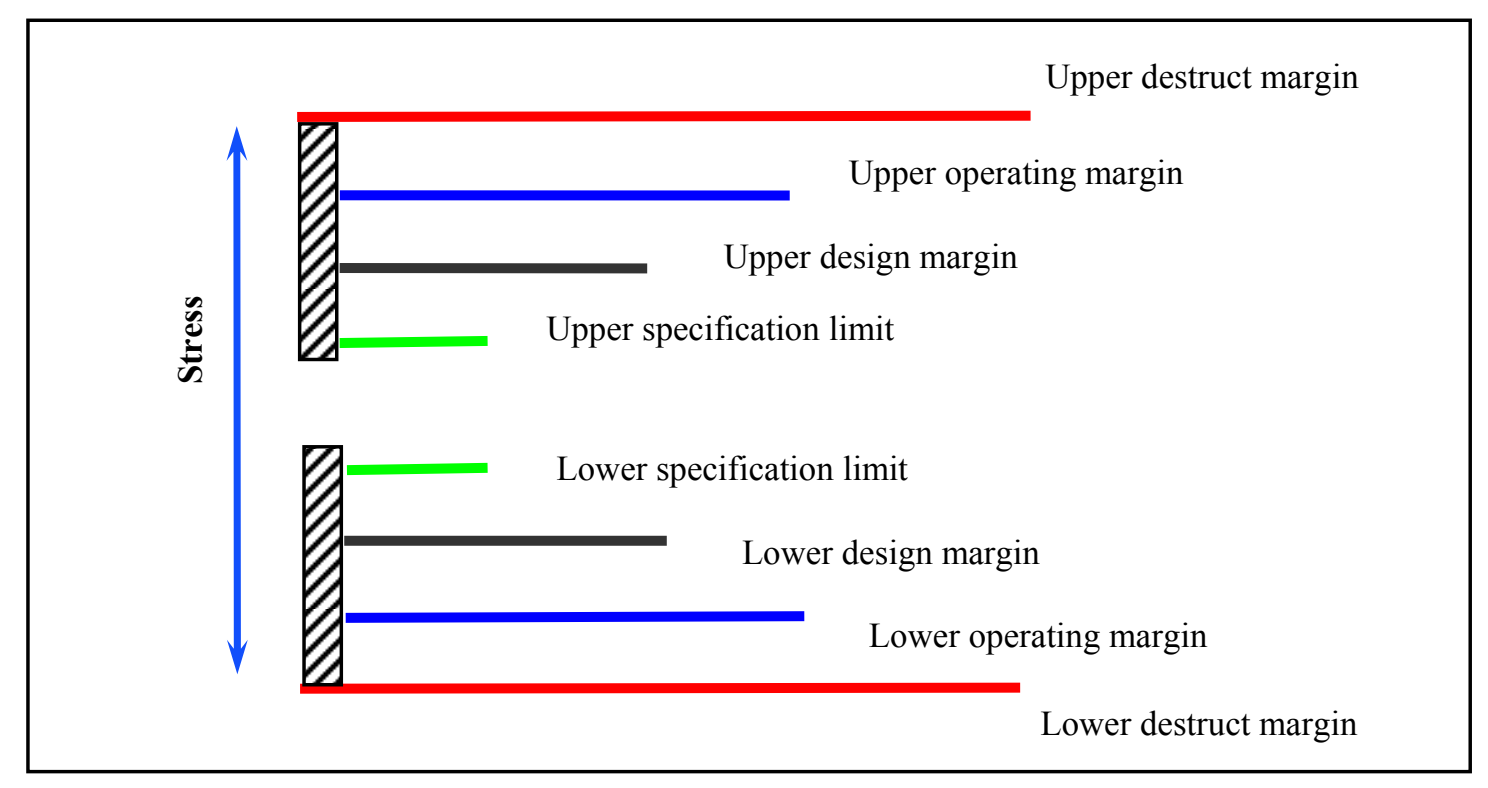

\subsection{Strength limits and margins}

Strength limits are obtained by following the methodologies of the highly accelerated life test (HALT). The purpose of HALT is to expose design weakness by iteratively subjecting the product to increasingly higher levels of stress and then learn what should be improved in

the upper operating limit and the upper destruct limit. The specification limits are provided by the manufacturer to limit the use conditions by the customer. The design limits are the stress conditions at which the product is designed to survive. The operational limits of the product are reached when the product can no longer function at the accelerated conditions due to a recoverable failure. The stress value at which the product fails permanently and catastrophically is identified as the destruct limit. Generally, large margins are desired between the operational and destruct limits, and between the actual performance stresses and the specification limits of the product, ensuring higher inherent reliability.

Accurate mean strength limits and margins can be identified only if sufficient numbers of samples are tested to reveal complete distribution characteristics. The strength limits obtained from HALT can be used in planning the accelerated test and screening conditions. The destruct limits can be used as the baseline for highly stress screening tests (HASS) during production level qualification. If the product demonstrates survivability well beyond its operational limits or the limits of screening equipment, then the search for destruct limits can be terminated.

For designs, the primary goal of the HALT is to place as much margin between the products specified or guaranteed operating limits and the observed operating limits during the HALT. Studies have consistently shown that products with generous performance margins between the specification and actual performance are inherently more reliable. The determination of the destruct limits are used to ensure that a sufficient margin exists between the operating and destruct limits to provide insight into how a product design/process can be improved and to establish a and margins diagram

the specific product. HALT is the first physical testing performed during the product qualification stage.

In product qualification, HALT can be used to identify the operational and destruct limits and margins, known as the 'strength limits' as shown in Figure 5. The limits include the upper and lower specification limit, the upper design margin, baseline for a production level highly accelerated stress screen (HASS). For some products, the search for the destruct limits may be aborted when the product exhibits survivability well beyond the previously determined operating limits or survivability at the limits of the screening equipment.

\subsection{Failure modes, mechanisms, and effects analysis (FMMEA)}

It is important to identify the critical failure mechanisms of a product induced by life-cycle loads. This will provide options for the selection of qualification tests. Only tests targeting the identified critical failure mechanisms should be selected as qualification tests. A cross-functional team (design, manufacturing, reliability, etc) will allow better identification of issues and criticality.

FMMEA is a methodology used to identify critical failure mechanisms. FMMEA utilizes the basic steps in developing a traditional FMEA in combination with knowledge of the physics of failure [23]. It then uses a life cycle profile to identify active stresses and to select the potential failure mechanisms. Knowledge of load type, level, and frequency combined with the failure site are used to prioritize failure mechanisms according to their severity and likelihood of occurrence. Figure 6 is a schematic diagram of FMMEA. FMMEA is based on understanding the relationships between product requirements and the physical characteristics of the product (and their variations in the production process), the interactions of product materials with loads (stresses at application conditions), and their influence on the product's susceptibility to failure. Potential failure mechanisms are determined based on appropriate available 


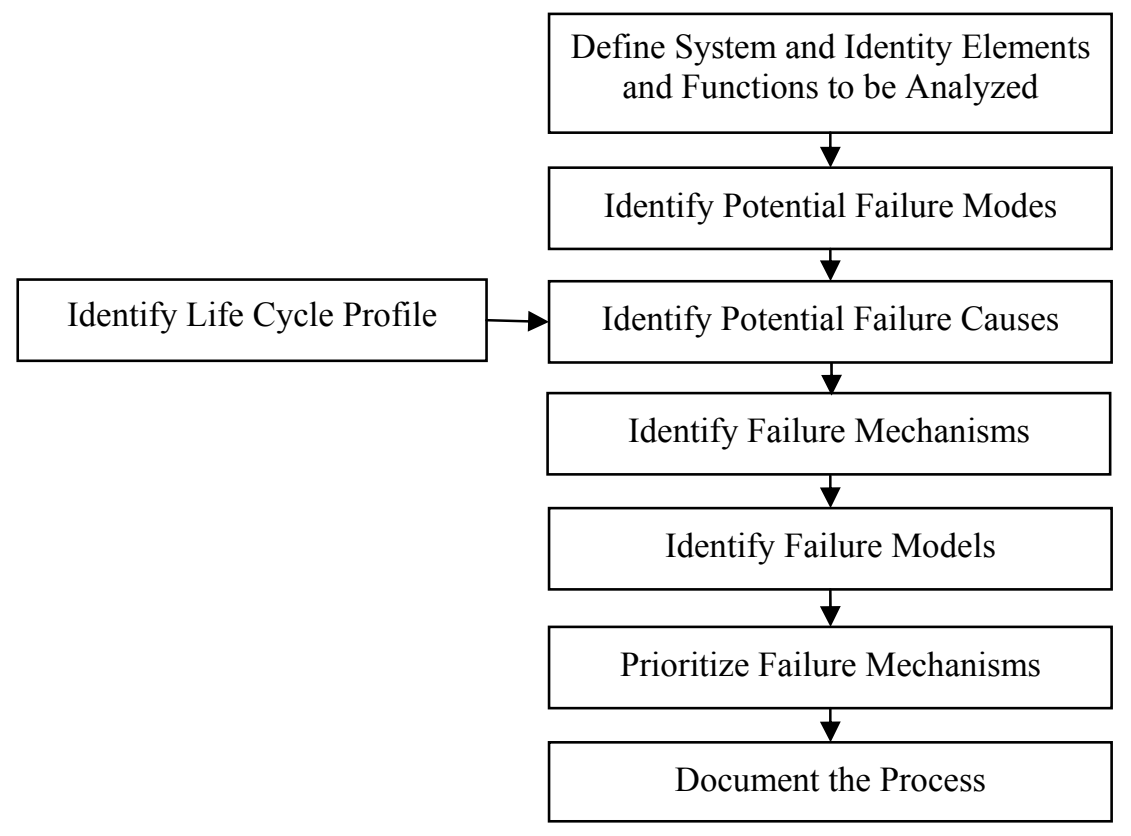

\section{Figure 6: FMMEA Methodology}

mechanisms corresponding to the material system, stresses, failure modes, and causes. FMMEA prioritizes the failure mechanisms based on their occurrence and severity to provide guidelines for determining the major operational stresses and environmental and operational parameters that must be either accounted for in the design or controlled. The high-priority failure mechanisms identified through the combination of occurrence and severity are the critical mechanisms. Critical failure mechanisms are the priority mechanisms considered in qualification tests. The failure sites, modes and causes associated with the critical failure mechanisms will provide information used to select the qualification test conditions.

The basic categories of failures are overstress (i.e., based on stress strength interference) and wear-out (i.e., based on damage accumulation); they are often identified through a mode that goes beyond performance tolerance (e.g., excessive propagation delays). Overstress and wearout failures generally result from irreversible material damage; however, some overstress failures can be caused by reversible material damage (e.g., elastic deformation).

Failure models are used as tools to assess failure propensity. In PoF models, the stresses and the various stress parameters and their relationships to materials, geometry, and product life are considered. Each potential failure mechanism is represented by one or more of the prevalent models. A model should provide repeatable results, reflect the variables and interactions that are causing failures, and predict the behavior of the product over the entire domain of its operational environment. This type of a model allows development of accelerated testing and may help to reduce the number of test runs. Many PoF models, such as the Arrhenius model, the Coffin-Mason model and the Steinberg model, exist for predicting the behavior of components and products. Different models have different associated assumptions, which limit their applications to specific ranges of conditions.

\subsection{Qualification requirements}

Qualification requirements are the quality and reliability properties of the product suited to demonstrate compliance to the application requirements [1]. Qualification requirements must define the objectives and contents of the qualification activities. Qualification requirements are derived from the application requirements of the customer or the application segments [24]. They are based on the application requirements specified by the customer including functional performance, application conditions and time (use condition profile), processing conditions, robustness against random external stresses and expected statistical reliability properties such as tolerable infant mortality failures. Different products have different application requirements, varying from benign environments and short term use to harsh environmental conditions and long term use. Qualification requirements should reflect the requirements of the application and should ensure that the qualified product can survive and perform its function reliably under the application conditions. Qualification requirements have also to be defined based on the life cycle load profile of the product. These loads include what the product experiences during its life cycle including manufacturing, assembly, storage, transportation and operation. Depending on the individual experience and biases of the person performing the evaluation, qualification can be classified by the following four levels, as shown in Figure 7.

Similarity: Similarity is the lowest form of reliability qualification. Processes, products and packages may be qualified by being similar to something that has previously been qualified to a higher level. This level is usually accomplished with an engineering argument based upon logic. For example, if a certain package style is capable of completing a series of environmental tests, then it is likely to pass similar tests, regardless of the design of the die. Therefore, many different integrated circuits (ICs) using the same type of package could be "qualified by similarity." This level is accomplished with the lowest amount of resources, but also carries the highest risk of omission since tests are not actually conducted for each qualification. 


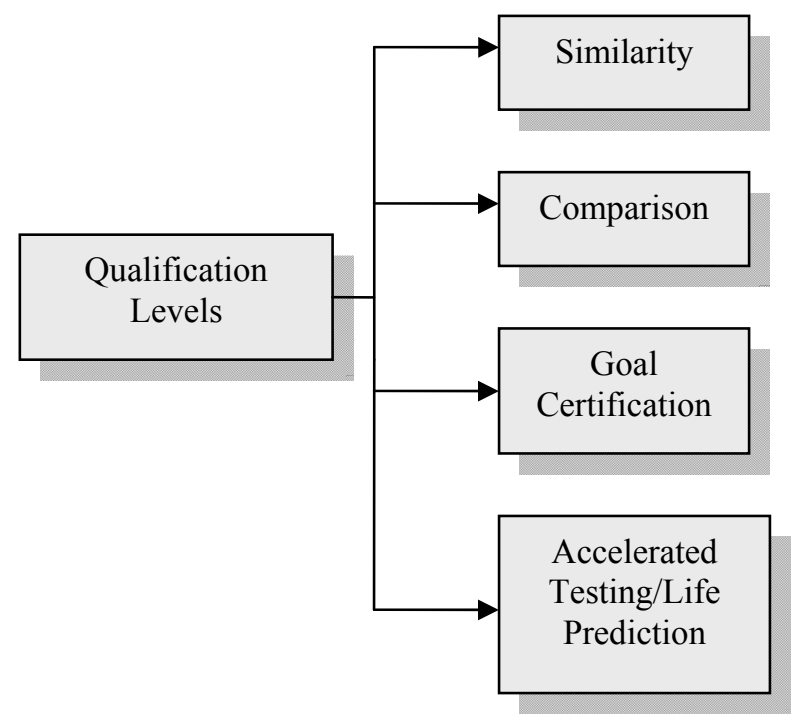

Figure 7: Qualification levels

Comparison: Comparison is the next level of qualification. It involves specific testing that compares results, but it does not necessarily meet goals of reliability for definable time periods. These tests generally collect attributes, not variables. For example, many packagerelated qualifications involve "standard" testing that does not have accepted acceleration factors that can be used to compare with normal life or expected use conditions, such as lead fatigue or temperature cycling. This is not to say that such testing doesn't have value, but favorable results usually can't be easily translated into specific statements about expected lifetimes. For example, what lifetime corresponds to 100 temperature cycles? We all know that it is good for a device to pass 100 temperature cycles without failure, but there is not necessarily an acceleration factor that can be used to translate the result to a failure rate ormedian life. Another form of comparison testing involves information about thermal performance, absolute maximum ratings, or other parameters that generally measure quality attributes that don't have direct correlation to reliability. In fact, results of comparison testing are often measured by attributes, not variables. In its most liberal form, qualification by comparison is achieved by matching results of previously performed tests or baselines

Goal certification: The goal certification level of qualification involves meeting specific goals involving life. For example, life tests confirming reliability performance during warranty periods or expected use periods may confirm the goals. This level is discerned from the comparison level since the results can be directly translated into longevity. This level is different from the next higher level since actual end-of-life need not be measured. For example, a one-year-long life test may be conducted without acceleration to cover an expected warranty period of 1 year. Knowledge of degradation modes, failure mechanisms, lifetimes, or acceleration factors will not likely be discovered. However, the data could be convincing in terms of knowing that products can survive reliably during the warranty period. Various goals can be certified using this level of qualification and the predetermined acceleration factors. This is the most common form of qualification, particularly in military specifications.
Accelerated testing/life prediction: The fourth and highest level of qualification involves accelerated testing and the time-to-failure measurement. Tests are conducted on the electronic package to simulate the life cycle environmental stresses under accelerated conditions for time compression. In this level of qualification, failure modes and mechanisms associated with failure sites are likely to be identified, and the median lifetime and reliability can be determined from the tests results and acceleration factors.

\subsection{Qualification Tests}

After the qualification requirements are defined, qualification tests will be conducted to examine whether the product can meet the requirements. However, before the tests are conducted, it is important to do test planning, including test condition selection and stress level selection. 3.6.1 Qualification test planning

Before qualification test planning is conducted, the qualification requirements have to be understood and converted to target values and tolerances that the product or the elements of the product have to meet. Relevant parameters and target values and tolerances are determined in order to meet or exceed the requirements. For example, a useful life requirement of a product has to be met by all its elements. By analysis, a weak element which has potential to fail earlier than others can be determined. Then a useful life requirement can be assigned to this element to make it meet a target useful life value and tolerance.

The qualification test conditions and stress levels are determined from the LCP of the product, FMMEA, experience from previous similar products and the qualification requirements. Target values and tolerances have to be met by the product or product elements under the test conditions selected in respect to the LCP [25]. Critical failure mechanisms, identified by FMMEA, associated with failure sites and failure modes will be considered in the qualification tests. When these failure mechanisms are combined with the reliability requirements, the targeted failure mechanisms in the qualification tests can be determined. If a failure mechanism identified to be critical in the FMMEA has no significant impact on the product's life within the reliability requirements, then this failure mechanism will 
not be considered in the qualification test. For example, if in the FMMEA, corrosion on printed circuit boards alone will cause failure to the product in 10 years, but the reliability requirement is that the product operates without failure for 5 years, then the corrosion won't be a concern in the qualification test.

The selection of the qualification test stress level should ensure that the stress will induce the same failure mechanism as it would under operating conditions without introducing any new failure mechanism. And the transformation between the qualification test results and the actual life under application conditions should be able to be realized based on acceleration factor.

The sample size selection is a critical issue in reliability qualification test planning. Sample sizes should be adequate for the characterization of the failure distribution. If the testing properties are systematically common to all products of a similar type: the sample size can be small. If the testing is for a small fraction of defective products, the sample size should be large corresponding to the small fraction to be determined.

Time to market and cost are two important constraints that have to be applied during the qualification test planning. Time to market is an important factor for a product to conquer more market share, especially for some new technologies. So it is better to compress the qualification testing time to be as short as possible in term of time to market. However, the qualification testing time depends on the testing conditions, stress level and sample size that are implemented during the testing. The testing conditions, stress levels and sample size have to be selected within allowable range based on the rules discussed in previous paragraphs. Cost is another factor that constrains the selection of stress types and stress levels. Higher stress, longer testing time or larger sample size may increase the cost of the qualification testing. So tradeoffs have to be made between time to market and cost without violating the underlying scientific rules that are governing the testing.

\subsubsection{Accelerated testing}

Many qualification tests are accelerated tests, since the higher level of stress can cause failures within a short period of time over the life cycle of the product. Accelerated testing allows for reduced test times by providing test conditions that "speed up" the evolution of failures, thus saving the time-to-market of a product. Accelerated testing involves measuring the performance of the test product at loads or stresses that are more severe than would normally be encountered, in order to enhance the damage accumulation rate within a reduced time period. The goal of such testing is to accelerate the timedependent failure mechanisms and the damage accumulation rate to reduce the time to failure. The failure mechanisms and modes in the accelerated environment must be the same as (or quantitatively correlated with) those observed under actual usage conditions, and it must be possible to quantitatively extrapolate from the accelerated environment to the usage environment with some reasonable degree of assurance.

There are two types of accelerated testing: qualitative accelerated testing and quantitative accelerated testing. In quantitative accelerated testing, the engineer is mostly interested in identifying failures and failure modes without attempting to make any predictions as to the product's life under normal use conditions. In quantitative accelerated life testing, the engineer is interested in predicting the life of the product at normal use conditions from data obtained in an accelerated life test.

Accelerated testing should be targeted at the critical failure mechanisms that have been determined. The load parameter that directly causes the time-dependent failure is selected as the acceleration parameter and is commonly called the accelerated load [26]. Common accelerated loads include thermal loads, such as temperature; chemical loads, such as corrosion; electrical loads, such as voltage; and mechanical loads, such as vibration. The accelerated test conditions may include a combination of these loads. Interpretation of the results for combined loads requires a quantitative understanding of their relative interactions and the contribution of each load to the overall damage. Failure due to a particular mechanism can be induced by several acceleration parameters, for example, corrosion can be accelerated by both temperature and humidity; and creep can be accelerated by both mechanical stress and temperature. Furthermore, a single acceleration stress can induce failure by several wearout mechanisms, simultaneously. For example, temperature can accelerate wearout damage accumulation not only by electromigration, but also by corrosion, creep and so on. Failure mechanisms that dominate under usual operating conditions may lose their dominance as the stress is elevated. Conversely, failure mechanisms that are dormant under normal use conditions may contribute to device failure under accelerated conditions. Thus, accelerated tests require careful planning in order to represent the actual usage environments and operating conditions without introducing extraneous failure mechanisms or nonrepresentative physical or material behavior. The degree of stress acceleration is usually controlled by an acceleration factor, defined as the ratio of the life under normal use conditions to that under the accelerated condition. The acceleration factor should be tailored to the hardware in question, and should be estimated from a functional relationship between the accelerated stress and reduced life, in terms of all the hardware parameters.

Once the dominant failure mechanisms are identified, it is necessary to select the appropriate acceleration load; to determine the test procedures and the stress levels; to determine the test method, such as constant stress acceleration or step-stress acceleration; to perform the tests; and to interpret the test data, which includes extrapolating the accelerated test results to normal operating conditions [26] [27]. The test results provide qualitative failure information for improving the hardware through design and/or process changes. Accelerated testing thus includes accelerated test planning and development, test vehicle characterization, accelerated life testing, and life assessment. Accelerated test planning and development is used to develop a test program that focuses on the potential failure mechanisms and modes that were identified in this phase, including design of the test matrix and test loads, analysis, design and preparation of the test vehicle, setting up the test facilities (e.g., test platforms, stress monitoring schemes, failure collection and postprocessing schemes), fixture design, effective sensor 
placement, data collection and post-processing schemes. Test vehicle characterization is used to identify the contribution of the environment on the test vehicle in accelerated life tests. Accelerated life testing is used to evaluate the vulnerability of the product to applied life cycle durability only if it is preceded by the steps discussed above. Without these steps, accelerated life testing can only provide comparison between alternate designs if the same failure mechanism is precipitated. Lastly, reliability assessment is used to provide a scientific and rational method to understand and extrapolate accelerated life testing failure data to estimate the life of the product in the field environment.

\subsection{Failure analysis and verification}

Detailed failure analysis of failed samples is a crucial step in the qualification and validation program. Without such analyses and feedback to the design team for corrective action, the purpose of the qualification program is defeated. In other words, it is not adequate simply to collect failure data. The key is to use the test results to provide insights into, and consequent control over, relevant failure mechanisms and to prevent them, cost effectively.

The purpose of doing failure analysis on samples which failed during the qualification testing is to verify that the failures were caused by failure mechanisms expected before the testing. No other failure mechanisms were induced during the testing. It insures the validity of the qualification testing that was intended to focus on specified failure mechanisms that would occur under application conditions.

If a product with new technologies was developed, the failure mechanisms before the testing may not be clear. The failure analysis is a chance to reveal the actual failure mechanisms that caused the failures. Thus the right PoF models can be selected to calculate the reliability of the product based on testing results in the following step.

\subsection{Quality and reliability assessment}

The reliability assessment is performed based on the accelerated test data and the PoF models. The reliability of the product is determined in terms of time-to-failure at the identified failure sites for a specific failure mechanism due to specific load condition. With the failure sites, stress inputs, and failure models, the reliability of a product is estimated and reported in terms of time to failure of the identified failure sites. Most failure models define time to failure under a specific loading condition. In the qualification test, the reliability of products is defined in order to meet the specified reliability requirement under qualification test conditions.

For most products, the life cycle profile consists of multiple loading conditions. As a result, methods for evaluating time to failure over multiple loading conditions must be derived. One approach is to cast the time to failure for a specific failure mechanisms in terms of the ratio of exposure time to the stress condition over time to failure for the stress condition. This ratio is often referred to as the damage ratio. If the exposure time is equivalent to the time to failure, then the ratio would equal to one. If one assumes that damage accumulates in a linear fashion, the damage ratios for the same failure site and mechanism can be added over multiple defined stress conditions. It is then assumed that once the accumulated damage ratio equals one, failure at the site would occur. For the same site and the same failure mechanism and for fixed duration load events, a specific damage ratio can be determined. For example, drop of a hand held device from a certain height may result in a loss of ten percent of the life of a solder interconnect. In this case, each drop will result in an increment of 0.1 damage ratio for the solder interconnect. For repetitive events, a damage rate may be established by the uses the appropriate failure models to estimate the number of events required to produce failure. The damage rate is then defined as one over the estimated number of survivable events. For example, if a failure model estimates that a solder interconnect can survive 2000 temperature cycles, then the damage rate per cycle is 0.0005 .

In general, time to failure data is obtained as a distribution for each failure site and failure mechanism. This distribution on time to failure is achieved by considering the inputs parameters to the failure models as distributions. In reality all dimensional and material properties are distributed about a nominal value as a result of variations in manufacturing. The same is true for the environmental loads. The physics of failure based reliability assessment allows for utilization of these natural variations in the reliability assessment. With the time to failure distribution on each site known, reliability can be evaluated in different metrics such as hazard rate, warranty return rate, or mean time to failure.

In addition to evaluating time to failure, the use of failure models allows for the examination of time to failure sensitivity to material, geometry, and life cycle profile. By considering the impact of the identified material and product geometries and loading conditions, the most influential parameters can be identified. This information can be used to improve design through closer attention to critical design parameters.

\section{Conclusions}

Qualification is an application-specific process involving the evaluation of the product with respect to its quality and reliability. The aim of the qualification process is to verify whether the product meets or exceeds reliability and quality requirements of the intended application.

Qualification plays an important role in the product development process. It occurs in different stages of the product development process with different purpose: design qualification, product qualification, quality assurance testing. Virtual qualification is based on PoF model predictions on the life of the design without any physical testing. Virtual qualification is relatively less expensive and less time-consuming than product qualification.

Product qualification involves physical tests on the manufacturer prototype, including highly accelerated life testing (HALT) to determine the strength limits of the product, and accelerated testing for reliability assessment. In product qualification, product configurations and materials and the life-cycle profile are captured as inputs to identify critical potential failure mechanisms associated with failure sites of the product under application conditions. Application requirements from customers are converted to determine the qualification requirements. In consideration of the life-cycle profile and qualification 
requirements, qualification test conditions, stress levels and sample size are selected to meet the function and reliability requirements. Accelerated tests are used in the qualification process due to the advantage of time compression. They accelerate the failure mechanisms in time due to the higher levels of stress than what the product would experience in the application environment. However, it should be ensured that only the failure mechanism under evaluation is being accelerated, while no other failure mechanisms are introduced. Quality and reliability of the product are assessed based the qualification test result. The assessment provides feedback to the design team to improve the product with tradeoffs.

\section{References}

[1] JEDEC, JEP 148, "Reliability Qualification of Semiconductor Devices Based on Physics of Failure Risk and Opportunity Assessment", April 2004.

[2] Pecht, M., Dasgupta, A., Evans, J., and Evans, J., Quality Conformance and Qualification of Microelectronic Packages and Interconnects, John Wiley \& Sons (New York, NY, 1994).

[3] IPC, IPC-9701, "Performance Test Methods and Qualification Requirements for Surface Mount Solder Attachments", January, 2002.

[4] Stark, B., and Kayali, S., "Qualification Testing Protocols for MEMS", NASA Document, http://parts.jpl.nasa.gov/docs/JPL\%20PUB\%20991J.pdf.

[5] Osterman, M., and Stadterman, T., "Failure Assessment Software for Circuit Card Assemblies", Proceedings of the IEEE Annual Reliability and Maintainability Symposium, (1999), pp. 269-276.

[6] Caruso, H., and Dasgupta, A., "A Fundamental Overview of Analytical Accelerated Testing Models", Journal of the Institute of Environmental Sciences, Vol. 41, No.1, January/February (1998), pp. 16-30.

[7] Hu, J., Barker, D., Dasgupta, A., and Arora, A., “The Role of Failure Mechanism Identification in Accelerated Testing", Journal of the Institute of Environmental Sciences, Vol. 36, No. 4, July (1993), pp. 39-45.

[8] Pecht, M., Radojcic, R., and Rao, G., Guidebook for Managing Silicon Chip Reliability, CRC Press (Boca Raton, FL, 1999).

[9] Cunningham, J., Valentin, R., Hillman, C., Dasgupta, A., and Osterman, M., "A Demonstration of Virtual Qualification for the Design of Electronic Hardware", Proceedings of the Institute of Environmental Sciences and Technology Meeting, Phoenix, AZ, April 2001.

[10] Cushing, M., Mortin, D., Stadterman, T., and Malhotra, A., "Comparison of Electronics-Reliability Assessment Approaches", IEEE Transactions on Reliability, Vol. 42, No. 4, December (1993), pp. 542-546.

[11] Larson, T., and Newell, J., "Test Philosophies for the New Millennium", Journal of the Institute of Environmental Sciences, Vol. 40, No. 3, May/June (1997), pp 22-27.
[12] GRCI Inc., "Reliability Assessment Process Improvement Demonstration (RAPID)", Contract No: F33615-96-D-5302, Delivery Order 041, Subtask: 3.3, prepared for ESC/DIT, 1998.

[13] Cunningham, J., Valentin, R., Hillman, C., Dasgupta, A., and Osterman, M., "A Demonstration of Virtual Qualification for the Design of Electronic Hardware," Proceedings of the Institute of Environmental Sciences and Technology Meeting, April 24 (2001).

[14] Pecht, M., Dasgupta, A., \& Barker, D., “The Reliability Physics Approach to Failure Prediction Modeling," Quality and Reliability Engineering International, Vol. 6, Iss. 4 (1990), pp. 276-273.

[15] Pecht, M., and Dasgupta, A., "Physics-of-Failure: An Approach to Reliable Product Development," Journal of the Institute of Environmental Sciences, Vol. 38, No. 5 (1995), pp. 30-34.

[16] McCluskey, P., Pecht, M., and Azarm, S., "Reducing Time-to-Market Using Virtual Qualification", Proceedings of the Institute of Environmental Sciences Conference, 1997, pp. 148-152.

[17] Dasgupta, A., and Pecht, M., "Material Failure Mechanisms and Damage Models," IEEE Transactions on Reliability, Vol. 40 (5), Dec. (1991), pp. 531-536.

[18] Pecht, M., Handbook of Electronic Package Design, Marcell Dekker Inc. (New York, NY, 1991).

[19] Pecht, M., Agarwal, R., McCluskey, P., Dishongh, T., Javadpour, S., and Mahajan, R., "Electronic Packaging Materials and their Properties", CRC Press, (Boca Raton, FL, 1999).

[20] Ganesan, S., and Pecht, M., Lead-free Electronics, Second Edition, John Wiley \& Sons, Inc. (New York, NY, 2006)

[21] Pecht, M., Nguyen, L., and Hakim, E., "Plastic Encapsulated Microelectronics: Materials, Processes, Quality, Reliability, and Applications", John Wiley Publishing Co., (New York, NY, 1995).

[22] Lall, P., Pecht, M., and Hakim, E., "Influence of Temperature on Microelectronics and System Reliability", CRC Press, (New York, 1997).

[23] Ganesan, S., Eveloy, V., Das, D., and Pecht, M., "Identification and Utilization of Failure Mechanisms to Enhance FMEA and FMECA," Proceedings of the IEEE Workshop on Accelerated Stress Testing and Reliability (ASTR), Austin, Texas, October 2-5 (2005).

[24] Cluff, D. K., and Osterman, M., "Defining Accelerated Test Requirements for PWBs: A PhysicsBased Approach," IPC Printed Circuits Expo, (Long Beach Convention Center, Long Beach, CA, March 24-28, 2002).

[25] Snook, I., Marshall, J., and Newman, R., "Physics of Failure as an Integrated Part of Design for Reliability", Proceedings of the IEEE Annual Reliability and Maintainability Symposium, (2003), pp. 46-54.

[26] Lall, P., Pecht, M., and Cushing, M., "A Physics-ofFailure (PoF) Approach to Addressing Device Reliability in Accelerated Testing," $5^{\text {th }}$ European Symposium on Reliability of Electron Devices, 
Failure Physics and Analysis, Glasgow, Scotland, October 4-7, (1994).

[27] Upadhyayula, K., and Dasgupta, A., "Physics-ofFailure Guidelines for Accelerated Qualification of Electronic Systems," Quality and Reliability Engineering International, Vol. 14, Iss. 6 (1998), pp. 433-447. 